\title{
Clinical Evaluation Of The Periodontal Condition During Fixed Orthodontic Treatment
}

\author{
Krisztina Martha, PhD \\ Department of Orthodontics, Faculty of Dental Medicine, University of \\ Medicine and Pharmacy Tirgu Mures, Romania \\ Irinel Panainte, Master Candidate \\ University of Medicine and Pharmacy Tirgu Mures, Romania
}

Alexandru Ogodescu, PhD

Department of Pediatric Dentistry, Faculty of Dental Medicine, Victor Babes

University of Medicine and Pharmacy, Timisoara, Romania

doi: 10.19044/esj.2016.v12n24p78 URL:http://dx.doi.org/10.19044/esj.2016.v12n24p78

\begin{abstract}
Throughout orthodontic treatment, oral hygiene is particularly important and must be rigorously controlled throughout therapeutic act. The aim of our clinical study was to examine the periodontal status of patients before, during and after orthodontic appliances are used. 50 patients were selected, plaque index, gingival index, modified papillae bleeding index and clinical probing depth was evaluated on anchorage teeth, separately those with orthodontic bands and tubes. One of the most common findings during this kind of treatment is gingival alteration in the posterior region. Our practical observations showed a different status of these alterations at patients wearing bands on the anchorage teeth versus those with buccal tubes. Using some of the indices which describes the periodontal status at adult and adolescent patients, we found out that adolescents present a higher plaque index, and the accumulation of dental plaque is more severe when bands are used in both categories. Another finding, which is very important in everyday practice is that gingival inflammation and plaque accumulation decrease one month after debonding.
\end{abstract}

Keywords: Orthodontic treatment, periodontal condition

\section{Introduction}

The accumulation of plaque is influenced by numerous factors such as diet, oral hygiene, fluoride exposure, saliva, the composition of the microbial flora and immunological factors. 
Moblie and fixed orthodontic appliances make oral hygiene difficult to maintain by creating retentive zones favoring the accumulation of dental plaque (Türkkahraman, 2005). Depending on the amount and composition of the biofilm, enamel demineralization, caries and gingival inflammation may occur - these are may occur well known consequences of orthodontic treatment in case of poor oral hygiene. (Sinclair, 1987). When mild gingival inflammation is present before fixed appliance therapy in young patients, there is a tendency for improvement of periodontal health during treatment. (Kohno, 2002)

A series of studies show that by replacing multiband fixed appliances with directly bonded brackets, plaque accumulation significantly decreases and the accumulation of plaque and gingival inflammation can be considered less severe. The use of directly bonded tubes on the labial surface of the anchorage teeth - usually first permanent molars - instead of orthodontic bands decreases plaque retention as well, but even so, gingival inflammation around anchorage teeth tend to appear in early phase of fixed orthodontic treatment when oral hygiene is not properly carried out.

The aim of our study was to find the answers for a series of clinical observations: (1) the accumulation of plaque and gingival inflammation are more severe in posterior areas; (2) these changes occur in a shorter time in teenagers than in adult patients; (3) the areas most sensitive to the occurrence of these inflammations are the molar interproximal areas. For this reason we assessed the gingival response to fixed orthodontic appliance therapy during treatment, indices were assessed in four examination times.

\section{Material and methods}

We selected fifteen patients out of those referred for orthodontic treatment to the Orthodontic Department of the University of Medicine and Pharmacy, Tîrgu Mureș, Romania. A written informed consent was obtained from each patient or their parents/legal representative before the first sampling. The protocol was reviewed and approved by the Ethical Committee of Scientific Research of the University of Medicine and Pharmacy Tirgu Mures, decision nr. 117/21.11.2013.

The subjects were divided into two groups according their age and both groups in two subgroups according to the type of attachment used (Table 1.):

- $\quad$ Group A - 30 subjects, mean age $14.7 \pm 1.3$ years

o A1 - 18 patients with orthodontic bands

o A2 - 12 patients with directly bonded tubes on the labial surface of the first permanent molars

- $\quad$ Group B - 20 subjects, mean age 21.1 2 2.8 years

o $\quad$ B1 - 8 patients with tubes 
o $\quad$ B2 - 12 patients with bands

$\begin{array}{ccc}\text { Group } & \text { A } & \text { B } \\ \text { Bonded } & 12 & 8 \\ \text { Banded } & 18 & 12\end{array}$

Table 1. Distribution of bonde and banded molars in both groups

Periodontal indexes were determined before the application of the attachments (T0), after which the patients were instructed in the use of mechanical methods plaque removal. Patients with clinical evidence of periodontal disease or those who have given general or local antibiotics three months before the first examination were excluded. The next evaluation was carried out one month after attachment placement (T1), six months after it (T2) and 1-3 months after attachment removal (T3).

The following clinical indicators were assessed:

- Plaque index (PI) both the thickness and extent of coloured plaque was measured on four areas of each tooth and average of the four values gave the value for each anchorage tooth.

- Gingival index (GI) determines the presence or absence of bleeding in the survey in four points for each tooth: disto-buccal, mesio-buccal, distooral and mesio-oral. Average values were calculated for each examined tooth. Using the mean values, the following distinction was made:

- $\quad 0.1$ - 1.0 - slight gingival inflammation

- $\quad 1.1$ - 2.0 - average gingival inflammation

- $\quad 2.1$ - 3.0 - severe gingival inflammation

- Modified papillae bleeding index - PBI - is a sensitive clinical indicator of inflammation of the gingiva and it is currently used to motivate patients in achieving good oral hygiene. Palpation of mesial and distal papillaewas performed, value 1 ment the presence of bleeding, 0 the absence of it.

- Clinical probing depth - CPD - its measurement was performed using a calibrated periodontal probe, driven parallel to the tooth axis to the base of the gingival attachment. The highest value for each gingival sulcus depth was recorded.

Regarding orthodontic treatment the same tube system was applied. NiTi archwires were used in the first 1-3 months of treatment and SS archwires in the second phase. Average treatment duration was 20.6 \pm 1.7 months.

\section{Results}

Mean values for the above mentioned indeces are presented in Table 2. - 5. for each subgroup. 
T0

T1

$1,22 \pm 0,44$

$1,63 \pm 0,74$

$1,87 \pm 0,78$

$3,44 \pm 0,88$

$2,56 \pm 0,72$
$\mathrm{T} 1$

$1,06 \pm 0,71$

$1.51 \pm 0,45$

$1,42 \pm 0,94$

$3,21 \pm 0,51$

$2,56 \pm 0,72$
Table 2. Values for A1 subgroup

$\mathrm{T} 2$

T3

$0,778 \pm 0,67$

$0,889 \pm 0,45$

$0,44 \pm 0,56$

$2,91 \pm 0,12$

T2

T3

$0,775 \pm 0,59$

$0,83 \pm 0,76$

$0,52 \pm 0,48$

$2.83 \pm 0,28$

Table 3. Values for A2 subgroup

T0

T1

$$
\begin{aligned}
& 0,82 \pm 0,47 \\
& 1,47 \pm 0,62 \\
& 1,18 \pm 0,97 \\
& 2,79 \pm 0,65
\end{aligned}
$$

$2,50 \pm 0.73$

Table 4. Values for B1 subgroup

T2

$0,78 \pm 0,55$

$1,87 \pm 0,57$

$1,76 \pm 0.69$

$3,11 \pm 0,49$

T3

$0,59 \pm 0,44$

$0,27 \pm 0,36$

$1,03 \pm 0,73$

$2,92 \pm 0,58$

PI

$0,69 \pm 0.63$

$0,74 \pm 0,54$

$0,50 \pm 0,32$

$0,64 \pm 0,16$

GI $\quad 0,57 \pm 0,73$

$1,16 \pm 0,69$

$1.65 \pm 0,7$

$0,19 \pm 0,30$

PBI

$0,85 \pm 0,64$

$0,89 \pm 0,78$

$1,28 \pm 0,96$

$0,96 \pm 0,38$

CPD

$2,50 \pm 0.73$

$2,76 \pm 0,65$

$2,79 \pm 0,87$

Table 5. Values for B2 subgroup 
At T0 examination time higher PI and GI values were obtained for teenage group comparing with adults, which shows a poorer oral hygiene due to the lack of motivation the examination characteristic of this age. Following IP values for the two groups in different phases of the orthodontic treatment higher values were obtained for teenagers at each examination, the highest values being recorded at the time $\mathrm{T} 2$.

Regarding the adult patients, the differences between the values obtained at all the four examination times were not significant, which shows good hygiene and a strong motivation before and during orthodontic treatment.

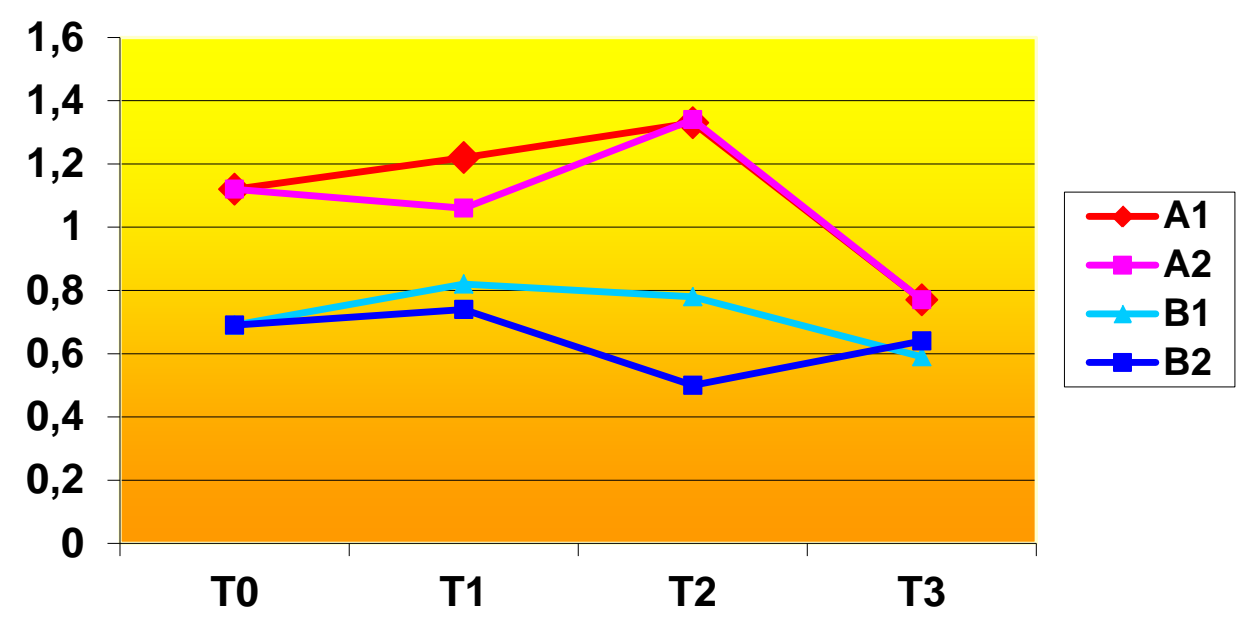

Fig.1. IP values for the four groups examined

PBI values were slightly higher for the adult group, the difference between the two groups for CPD was not significant statistically.

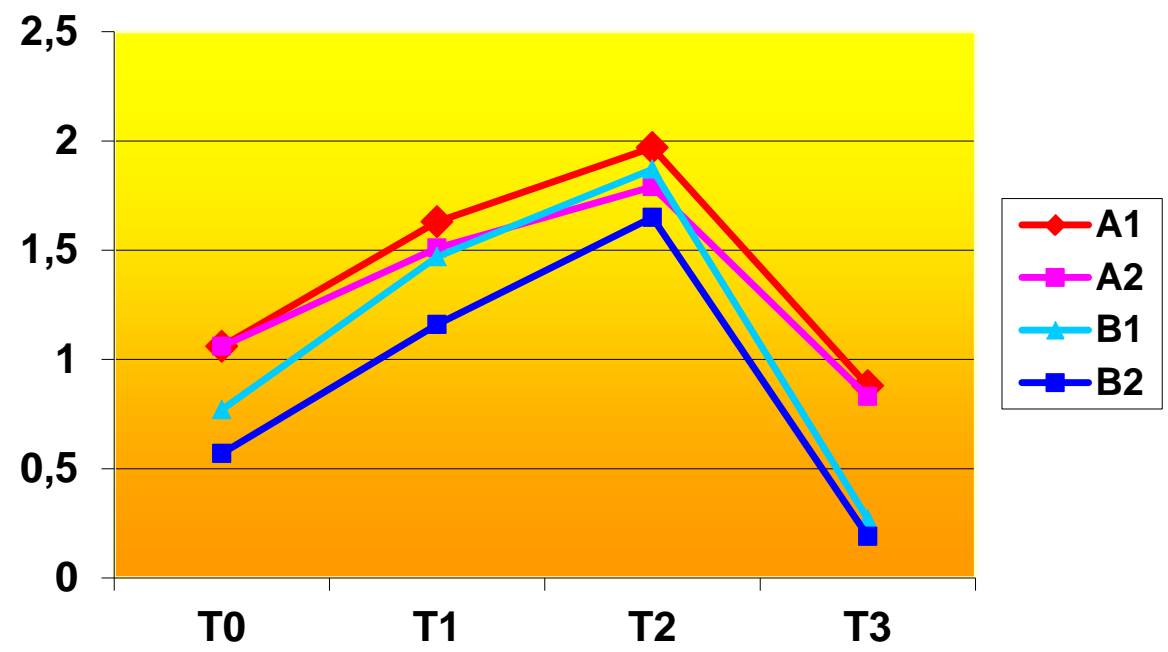

Fig.2. IIG values for the four groups examined 
The indices of gingival inflammation (GI) and of bleeding (PBI) increase quite pronounced in the first months of treatment in all patients, reaching relatively high values after 6 months of treatment. It is interesting to note that after the removal of orthodontic appliances these indeces showed values below those obtained before appliance bonding. The dynamic of CPD values changing showed similar trends to those described above.

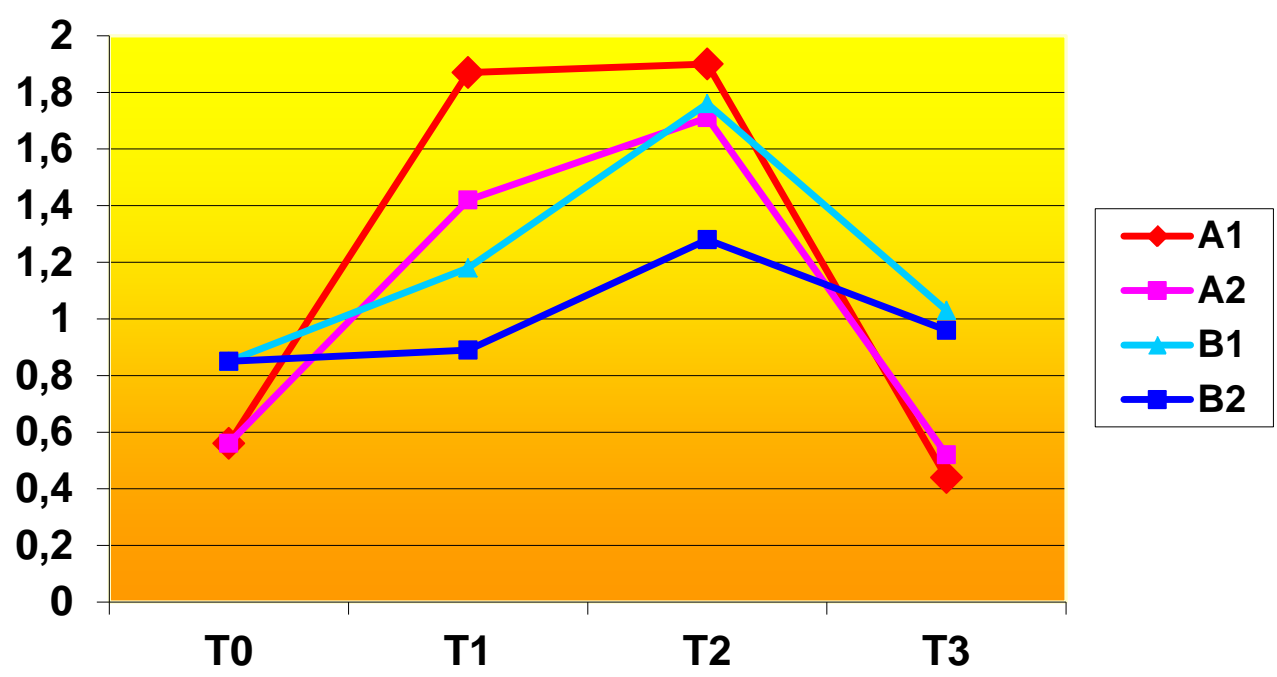

Fig. 3. PBI values for the four groups examined

Comparing all the values obtained in the various subgroups we observed lower values for all indexes regardless of age in patients with directly bonded tubes compared with the ones where bands were used.

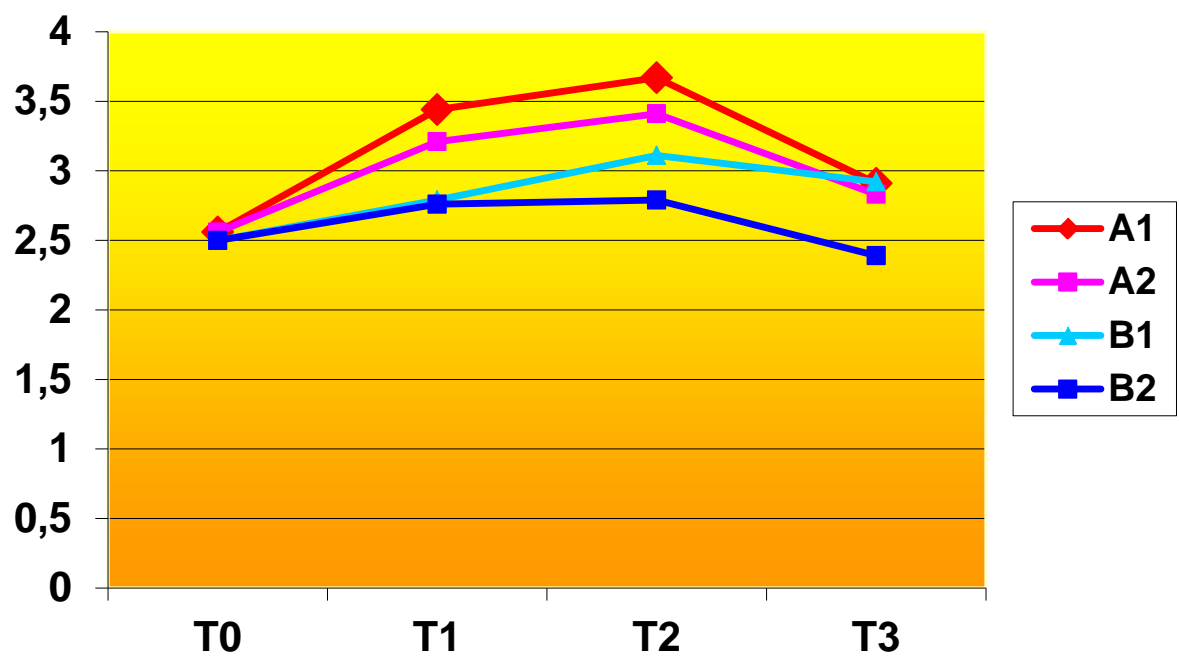

Fig. 4. ACS values for the four groups studied 
The results of this study confirm some hypotheses presented in the introduction: (1) the accumulation of plaque is more pronounced in patients in their teens, compared to adults; (2) molars with orthodontic bands show an increased accumulation of plaque and gingival inflammation is more pronounced; (3) even if these attachments are worn for a long time, this does not influence periodontal status of the teeth in question.

It is becoming increasingly clear that age plays an important role in maintaining oral hygiene. During adolescence, young people tend to neglect their oral health are not motivated enough or careful in this regard. Often the establishment of an orthodontic treatment tends to improve the situation, arches and default alignment-enhancing appearance aspect makes young people to be more demanding in oro-dental health maintenance.

Similar findings were also reported by Boyd et al. (1992). Comparing the periodontal responses in orthodontic treatment for adolescents and adults, the author points out that the accumulation of plaque and gingival inflammation is more severe in adolescents both before and during fixed orthodontic treatment, the differences between the values of periodontal indices were higher after appliance removal. The same longitudinal study shows that the areas most affected are the interproximal ones, increased plaque accumulation and gingival inflammation was found in the molar region and on molars with orthodontic bands compared with the ones with directly bonded tubes. Gingival attachment loss was found to be greater on upper molars with bands.

Examining two years after treatment 51 patients with abnormal Angle II / 1 solved by extracting four premolars and comparing the depth of periodontal pockets, attachment loss and height of the clinical crown with values obtained from a control group of 54 untreated patients orthodontic, Zachrisson (1972) shows that the values obtained were significantly higher in patients after treatment with fixed appliances. Even if the values obtained from both groups are not worrying, the author wishes to emphasize that malocclusions like increased overjet or open bite are factors that can not be incriminated in extent or severity of gingivitis. Posttreatment periodontal changes can be explained by high orthodontic forces beyond biological limits and that with occlusal traumas could be considered factors which may lead to development of periodontal pockets.

Given the specific structure of bone and periodontium in lower frontal areas, gingival recession occurs more frequently in this region. The risc of recession is greater when orthodontic treatment involves an excessive protrusion of the front teeth in order to create the necessary space to solve frontal crowding. If you can not identify other factors that may contribute to periodontal inflammatory processes, controlled protrusion of these teeth can 
be accomplished without jeopardizing periodontal health in this area. (Allais, Melsen, 2003)

In a study on periodontal status of patients referred to orthodontic treatment (Onyeaso, 2003) a thorough periodontal examination was performed before appliance bonding. 145 patients were examined, 91.8\% were aged under $25,0.7 \%$ showed signs of periodontal destruction. This low percentage shows relatively low frequency of periodontal disease in adolescents and young adults.

Davies et al. (1991) in their study on the effect of orthodontic appliances on plaque accumulation and gingival condition have shown that periodontal and gingival health is a consequence of oral hygiene more than dental alignment.

Although orthodontic treatments are long term, Sadowsky and Bego (1981) shows the fixed devices have no significant effect on the periodontal health of mucous tissues.

\section{Conclusion}

- $\quad$ Orthodontic bands and tubes will create retentive zones which will make oral hygiene methods difficult to proceed

- $\quad$ Adolescents present more important plaque accumulation than adults

- The accumulation of plaque is more severe on teeth where orthodontic bands were used, compared with directly bonded tubes

- $\quad$ The accumulation of plaque in adolescents increases considerably during the first month of treatment with fixed appliances. In adults this accumulation of plaque occurs later in the second part of the treatment

- $\quad$ Gingival inflammatory processes occur in the first three months of orthodontic treatment in children and adult patients also

- $\quad$ Both the accumulation of plaque and gingival inflammation decreases after removal of the appliance, periodontal status improves compared to the situation before the application of the fixed orthodontic appliance.

\section{Acknowledgements}

This research is supported by the University of Medicine and Pharmacy, Tîrgu Mureș, Romania, Internal Competition of Research Grants, grant number 35/11.12.2013.

\section{References:}

Allais D, Birte Melsen (2003) - Does labial movement of lower incisors influence the level of the gingival margin? A case-control study of adult orthodontic patients. European J Orthod. 25:343-352 
Boersma JG, van der Veen MH, Lagerweij MD, Bokhout B, Prahl-Andersen B (2005) - Caries Prevalence Measured with QLF after Treatment with Fixed Orthodontic Appliances: Influencing Factors. Caries Res., 39:41-47 Bogren A, Teles R, Torresyap G, Haffajee AD, Socransky SS, Lindhe J, Wennström JL (2007) - A three-year prospective study of adult subjects with gingivitis. I: clinical periodontal parameters. J Clin Periodontol, 34: 1-6.

Boyd RL, Baumrind S (1992) - Periodontal cosiderations in the use of bonds or bands on molars in adolescents and adults. Angle Orthodontist., 62(2): 117-125

Closs LQ, Branco P, Rizzatto SD, Raveli DB, Rösing KK (2007) - Gingival margin alterations and the pre-orthodontic treatment amount of keratinized gingival. Braz Oral Res.,21(1):58-63

Crossner CG , Unell L (2007) - A longitudinal study of dental health from the age of 14 to 41 . Swed Dent J., 31 (2):65-74

Davies TM, Shaw WC, Worthington HV, Addy M, Dummer P (1991) - The effect of orthodontic treatment on plaque and gingivitis. Am J Orthod Dentofacial Orthop.,99:155-62

Diamanti-Kipioti A, Gusberti FA, Lang NP (1987) - Clinical and microbiological effects of fixed orthodontic appliances. Journal of Clinical Periodontology.,14 (6): 326-333.

Diedrich P, Rudzki-Janson I, Wehrbein H, Fritz U (2001) - Effects of orthodontic bands on marginal periodontal tissues. A histologic study on two human specimens. J Orofac Orthop. Mar;62(2):146-56

Djeu G,Catherine Hayes, Zawaideh S (2002) - Correlation Between Mandibular Central Incisor Proclination and Gingival Recession During Fixed Appliance Therapy. Angle Orthod. ,72:238-245

Fioretti F, Opsahl Vital S, Marchal Sixou C, Sixou M, Chaussain Miller C, Lasfargues JJ (2007) - Caries risk assessment by different approaches before fixed orthodontic treatment. European Cells and Materials., 13(1): 42

Kohno T, Matsumoto Y, Kanno Z, Warita H, Soma K (2002) Experimental tooth movement under light orthodontic forces: rates of tooth movement and changes of the periodontium. J Orthod., 29: 129-135

Kounoupis V, Tsalikis L, Dangalis P (2006) - Clinical and microbiological changes during orthodontic treatment and after appliance removal. Hellenic Orthodontic Review., 9(2): 103-121

Marsh PD (2004) - Dental Plaque as a Microbial Biofilm. Caries Res., 38:204-211

Müller HP, Heinecke A (2002) - The influence of gingival dimensions on bleeding upon probing in young adults with plaque-induced gingivitis. Clin Oral Investig., 6(2):69-74.

Nanci A, Bosshardt DD (2006) - Structure of periodontal tissues in health and disease. Periodontology 2000., 40: 11-28 
Ngom PI, Diagne F, Benoist HM, Thiam F (2005) - Intraarch and Interarch Relationships of the Anterior Teeth and Periodontal Conditions. Angle Orthod, 76(2): 236-242.

Onyeaso CO. (2003) An assessment of relationship between self-esteem, orthodontic concern, and Dental Aesthetic Index (DAI) scores among secondary school students in Ibadan, Nigeria. Int Dent J.;53:79-84.

Peralles P, Borges Viana AP, Luiz da Rocha A, Ramôa Pires F (2006) Gingival and alveolar hyperplastic reactive lesions: clinicopathological study of 90 cases . Brazilian Journal of Oral Sciences., 5(18): 1085-1089

Sallum EJ, Nouer DF, Klein MI, Gonçalves RB, Machion L (2004) Clinical and microbiologic changes after removal of orthodontic appliances. AJODO, 126(3): 363-366

Sinclair PM, Berry CW, Bennett CL, Israelson H (1987) - Changes in Gingiva and Gingival Flora with Bonding and Banding. Angle Orthod, 57(4): 271-278

Trombelli L, Farina R, Manfrini R, Tatakis DN (2004) - Modulation of Clinical Expression of Plaque-induced Gingivitis: Effect of Incisor Crown Form. J Dent Res., 83(9):728-731

Türkkaahraraman H, Sayin o, Bozkurt Y, Yetkin Z, Kaya S (2005) Archwire Ligation Technique, Microbial Colonization and Periodontal Status in Orthodontically Treated Patients. Angle Orthod, 75(2):227-232 Zachrisson BU (1972) - Gingival condition associated with orthodontic treatment. Angle Orthod, 42(2): 352-357 